\title{
Indoor fire in a nursing home: evaluation of the medical response to a mass casualty incident based on a standardized protocol
}

\author{
S. W. Koning $\cdot$ P. M. Ellerbroek $\cdot$ L. P. H. Leenen
}

Received: 26 July 2014/ Accepted: 1 September 2014/Published online: 25 September 2014

(C) Springer-Verlag Berlin Heidelberg 2014

\begin{abstract}
Purpose This retrospective study reports the outcome of a mass casualty incident (MCI) caused by a fire in a nursing home.

Methods Data from the medical charts and registration system of the Major Incident Hospital (MIH) and ambulance service were analyzed. The evaluation reports from the MIH and an independent research institute were used. The protocol for reports from major accidents and disaster was used to standardize the reporting [Lennquist, in Int $\mathbf{J}$ Disaster Med 1(1):79-86, 2003].

Results The emergency services were quickly at the scene. The different levels of pre-hospital management performed a tight coordination. However, miscommunication led to confusion in the registration and tracking of patients. In total, 49 persons needed medical treatment, 46 were treated in the MIH. Because of (possible) inhalation injury nine patients needed mechanical ventilation and nine patients were hospitalized to exclude delayed onset of pulmonary symptoms. No incident related deaths occurred. The intensive care unit of the MIH was initially understaffed despite the efforts of the automated calling system and switchboard operators. The handwritten registration of
\end{abstract}

S. W. Koning $(\bowtie) \cdot$ P. M. Ellerbroek · L. P. H. Leenen

Major Incident Hospital, University Medical Center Utrecht, Utrecht, The Netherlands

e-mail: swkoning@gmail.com

P. M. Ellerbroek

Department of Internal medicine and infectious diseases,

University Medical Center Utrecht, Utrecht, The Netherlands

L. P. H. Leenen

Department of Surgery and traumatology, University Medical Center Utrecht, Utrecht, The Netherlands incoming staff was incomplete and should be performed digitally. Some staff members were unfamiliar with the MIH procedures. The medical chart appeared too extensive. Miscommunication between chain partners resulted in the delayed sharing of (semi) medical information.

Conclusion The different levels of incident managers performed a tight coordination. The MIH demonstrated its potency to provide emergency care for 46 patients and 9 intubated patients. No deaths or persistent disabilities occurred. Areas of improvement were recognized both in the pre-hospital as the hospital phase.

Keywords Disasters [MESH] - Disaster medicine [MESH] - Evaluation studies [MESH] - Fires [MESH] · Mass casualty incidents [MESH] · Major Incident Hospital

\author{
Abbreviations \\ COPI Local incident commanders \\ GHOR Regional organization for medical assistance \\ during accidents and disasters \\ GRIP Coordinated regional procedure for incidents \\ control \\ ICU Intensive care unit \\ MCI Mass casualty incident \\ MIH Major Incident Hospital \\ PRMD Protocol for reports from major accidents and \\ disasters \\ UMCU University Medical Center Utrecht
}

\section{Introduction}

During a mass casualty incident (MCI) the medical need exceeds the response capabilities in the affected area [2-5]. 
To treat the overwhelming number of casualties this calls for a different approach from "do the most for one" to "do the best for most" [5].

Field triage is the most important parameter to classify patients who need immediate help and to establish to which hospital patients should be transported. The principles of triage have been described previously [4, 6-8]. In short, four triage classes are discerned: the $\mathrm{T} 1$ patients are those in mortal danger, $\mathrm{T} 2$ patients are severely injured but can wait for a limited time, whereas T3 patients are those least injured and $\mathrm{T} 4$ patients are those with a low probability of survival and those who have died. After triage and primary treatment, patients are transported to hospitals with different levels of trauma care based upon triage classification and nature of injuries. In the Netherlands, the joint emergency dispatch center and the medical assistance during accidents and disasters (GHOR) have predetermined the maximum (surge) capacity per hospital during a MCI.

This report analyzes the characteristics of a MCI that occurred after a fire spread rapidly through a nursing home on the morning of June 27th 2011. Due to the unusual spread of the fire the evacuation took longer than expected, which resulted in prolonged exposure to smoke and soot by the entrapped victims. The majority of patients were primarily treated in the Major Incident Hospital (MIH) in Utrecht which is part of the University Medical Centre of Utrecht (UMCU) in the Netherlands. The role and the goals of the MIH in the Netherlands have been described previously $[9,10]$. In short, the MIH is a reserved hospital that can become operational within $30 \mathrm{~min}$ after activation. It is part of the hospital, the regional and if needed the national disaster plan in the Netherlands and is a collaboration of the Central Military Hospital and the University Medical Centre of Utrecht. The MIH has 200 available beds, of which 12 intensive care unit (ICU) beds, a medium care ward with 25 beds and a large low care ward. The MIH has its own radiology department, three fully equipped operating rooms and sixteen mechanical ventilators.

After arrival in the MIH, patients always follow a predefined route. An experienced surgeon performs a quick retriage when patients are unloaded from the ambulances in the ambulance bay. Patients are then registered by the previous described Patient Barcode Registration System $[11,12]$ which allows patient tracking trough the MIH. From there, T1 and T2 patients will be transported to two corresponding triage bays ( $\mathrm{T} 1$ and $\mathrm{T} 2$ bay) which are staffed by physicians and nurses from the surgery, emergency medicine and anesthesiology department. After 15 min of primary survey, the patients will be transported to the operation room, the CAT scan or directly to the ICU, medium care, low care, where secondary survey and further treatment are performed. All T3 victims will be directly transported to the low care unit of the MIH.
The MIH is staffed by personnel from the UMCU and the Central Military Hospital. The MIH has a small group of key staff members (nurses, physicians and supporting staff) who follow monthly trainings and know the MIH and its procedures in detail. During an activation of the MIH these staff members perform coordinating roles. Training sessions are monthly offered to personnel of the UMCU and the Central Military Hospital to become familiar with the MIH materials and procedures. During an activation of the MIH, key staff members will be warned by the primary automated calling system. The surgeon on call can decided to activate the secondary automated calling system, triggering more staffs from different medical specialties.

All previous deployments of the MIH have been described previously $[9,10]$. The MIH has been used most frequently to give medical care to repatriated wounded Dutch soldiers and Dutch citizens who have suffered a MCI or disaster in the Netherlands or abroad.

The current report analyzes the fire incident using the protocol for reports from major accidents and disaster (PRMD) [1] to standardize reporting on MCIs.

\section{Materials and methods}

All available patient and logistic data from the incident were retrieved from the MIH hospital information systems (written and electronic charts), the Patient Barcode Registration System, the evaluation report from the MIH and the report from the Institute for Safety and Crisis Management which is an independent research agency (Title: Brand in zorgcentrum De Geinsche Hof: Evaluatie van de gebeurtenissen en de aanpak van de brand op 27 juni 2011). Additionally, the data collected by the Regional Ambulance Service Utrecht were analyzed.

The following demographic and patient data were collected: age, gender, types of injuries and treatment. A (possible) respiratory tract injury was classified if one of the following criteria was present: carbonaceous sputum, facial burns/singed hairs, signs of distress or hypoxemia, hoarseness, distinctive breath sounds, oropharynx or nasopharynx swelling or inability to swallow [13].

The statistical software SPSS 20.0 was used for recordkeeping and statistical analyses. The incident was analyzed using the PRMD [1]. We used the standardized tables from the PRMD with liberal variation.

\section{Results}

Summary of the scenario/hazard, description of accident

On Monday 27th of June 2011 at 07:55 a.m., a fire started during renovation of the roof of a nursing home and spread 
through the building. Due to the materials used, a large amount of smoke and soot developed which entered through the nursing home to the air-conditioning system. The inflow of smoke and soot alerted nearly all fire detectors simultaneously. The location manager ordered a complete evacuation, however, at that time the assigned evacuation routes had already been blocked through the exceptional spread of the fire. Thus all departments were eventually cleared by construction workers, nursing staff and bystanders, some patients via the roof of a side building. On the day of the fire, the nursing home had 138 inhabitants, which all had special physical and/or psychiatric needs. Almost all inhabitants were dependent on wheelchairs or other walking aids and sixteen inhabitants were bedridden. There were 49 staff members of the nursing home working at that time of the day.

Pre-hospital availability and alert (Table 1)

The fire department was alerted by an automatic warning system at 07:57 a.m. The first fire engine arrived after 7 min (08:04 a.m.) and concluded that more emergency services were needed. Through the joint emergency dispatch center the first ambulance was called to the scene and arrived $21 \mathrm{~min}$ later (08:18 a.m.). The first ambulance on the scene called in at least 10 other ambulances. At the end of the day 23 ambulances were used for the transportation of victims.
Hospital resources availability and alert

The joint emergency center requested the MIH to activate the hospital disaster plan. The MIH, a reserved hospital had 200 beds available, 12 intensive care beds, 16 ventilators and three operation rooms. The hospital closest to the disaster had activated their hospital disaster plan. All other hospitals in the regional trauma system were not called upon.

\section{Utilization of transport resources (Table 2)}

A total of 49 patients were treated in a hospital. Twentythree ambulances transported 47 patients to two different hospitals. The MIH treated 46 patients of which 44 were transported by ambulances and two had other means of transportation. Three patients were transported by ambulance from the incident site to the hospital closest to the incident.

The first patient was transported within $45 \mathrm{~min}$ to the nearest hospital in Nieuwegein. The second MCI patient was transported to the MIH after $48 \mathrm{~min}$. All patients were evacuated within $5 \mathrm{~h}$ (279 $\mathrm{min}$ ) after the initial alarm. Two patients reported themselves later that day to the emergency department of the UMCU and were transferred to the adjoining MIH. Additional ambulances were used to transport unharmed clients and staff of the nursing home to nearby sheltering homes. Eight ambulances from three

Table 1 Pre-hospital available and alerted

\begin{tabular}{|c|c|c|c|c|c|}
\hline Unit & Size/type & Distance & Alerted & Alerted (time) & First unit on scene \\
\hline \multicolumn{3}{|l|}{ Fire department } & \multicolumn{3}{|c|}{ Yes, automatic } \\
\hline First notification & & & & 07:57 & - \\
\hline First unit & Fire engine & NA & & $07: 59$ & 08:04 \\
\hline \multicolumn{3}{|l|}{ Ambulance } & \multicolumn{3}{|l|}{ Yes } \\
\hline ADC & NA & NA & & & \\
\hline First ambulance primary location & UN & UN & & $08: 10$ & $08: 18$ \\
\hline First ambulance secondary location & UN & UN & & $08: 12$ & $08: 31$ \\
\hline Number of ambulances & 23 & UN & Yes & $8: 19$ & \\
\hline \multicolumn{6}{|c|}{ Pre-hospital medical team and helicopter not alerted } \\
\hline GHOR & & UN & Yes & & \\
\hline First SMO & 1 & UN & & 08:14 & 08:26 \\
\hline Second SMO & 1 & UN & & 08:50 & 09:09 \\
\hline $\mathrm{MCOC}^{\mathrm{e}}$ & 1 & UN & & $\mathrm{UN}$ & 09:11 \\
\hline Medical loading officer & 1 & UN & & UN & 09:11 \\
\hline Police & \multicolumn{5}{|c|}{ Alerted and functioning, this lies beyond the scope of this article } \\
\hline Military & \multicolumn{5}{|c|}{$\begin{array}{l}\text { Due to the joint collaboration of the Central Military Hospital and UMCU, the military was alerted } \\
\text { through the mere activation of the MIH. Coincidentally the Minister of Defense was present in the } \\
\text { Central Military Hospital during activation }\end{array}$} \\
\hline
\end{tabular}

$A D C$ ambulance dispatch center, GHOR medical assistance during accidents and disasters, SMO senior medical officer, $M C O C$ medical chief of command, $U N$ unknown, $N A$ not applicable 
Table 2 Utilization of transport resources

$U N$ unknown, $N A$ not applicable

${ }^{\text {a }}$ One ambulance transported one patient to Hospital A and one to the MIH

\begin{tabular}{lllllll}
\hline Unit & $\begin{array}{l}\text { Number } \\
\text { alerted }\end{array}$ & $\begin{array}{l}\text { Number } \\
\text { used in } \\
\text { action }\end{array}$ & $\begin{array}{l}\text { Number of } \\
\text { deployments }\end{array}$ & $\begin{array}{l}\text { Number of } \\
\text { transported } \\
\text { patients }\end{array}$ & $\begin{array}{l}\text { First unit } \\
\text { departs with } \\
\text { patient }\end{array}$ & $\begin{array}{l}\text { Last unit } \\
\text { departs with } \\
\text { patient }\end{array}$ \\
\hline Ambulance & 29 & $23^{\text {a }}$ & 39 & 47 & $08: 42$ & 11.56 \\
Supportive & 6 & 6 & 6 & NA & NA & NA \\
Hospital A & - & $3^{\text {a }}$ & 3 & 3 & $8: 42$ & $11: 56$ \\
Major Incident Hospital & - & $21^{\text {a }}$ & 36 & 44 & $8: 45$ & $10: 14$ \\
Private car & & 2 & 2 & 2 & UN & UN \\
\hline
\end{tabular}

neighboring safety regions assisted the regional ambulance service. The total number of ambulances was sufficient to quickly extract the $\mathrm{T} 1$ and $\mathrm{T} 2$ patients. The medical assistance during accidents and disasters (GHOR) had arranged additional support staff and materials. The joint emergency dispatch center had requested a free highway lane between the incident and the MIH at the executive body of the Dutch Ministry of Infrastructure and the Environment. This unfortunately resulted in a barrier for some hospital staff to reach the MIH in the simultaneously evolving rush hour.

Hospital alert plan and response (Table 3)

The primary calling system was activated at 08:45 a.m. to alert all key staff members. The alert did not reach all of them on their mobile phones because part of the personnel was already at work and others were commuting to or from work. Switchboard operators of the UMCU additionally phoned all key staff members, wards and heads of department of the UMCU which took a considerable amount of time. The secondary calling system was not activated because the primary calling system combined with the efforts of the switchboard operators had alerted enough key staff members except for IC personnel. Three minutes before the first patient arrived (08:50 a.m.) all primary triage personnel were present. However, IC personnel were still on their way to the MIH when the first T1 patient was intubated. As mentioned before, some incoming staff had been hindered to reach the MIH due to the blocked highway lane.

Some of the staff members of the UMCU appeared to be unfamiliar with the MIH and its procedures. However, the key MIH staff members made it possible for all other personnel to perform their work in the MIH.

The hospital closest to the incident anticipated an increased patient flow and activated its own disaster plan. This plan was quickly revoked when it became apparent that all patients were transported to the MIH.
Coordination and command (Table 4)

After 13 min (08:12 a.m.) the joint emergency dispatch center had upgraded the incident to a Coordinated Regional Procedure for Incidents Control 1 (GRIP-1) by which a centralized level of disaster management became operational. The local incident commander team (COPI) was formed by the commanders of the firefighters, police and ambulance service, an information manager, a plotter and spokespersons of the nursing home. This multidisciplinary meeting addressed potential bottlenecks (status of the fire, evacuation routes, location of the forwarded medical post, location of sheltering the unharmed, registration and identification of transported clients, initiating psycho-social aftercare). The COPI agreed that unharmed clients were to be transferred only once to a primary shelter location. Busses were used to transport unharmed clients to the primary shelter location of the nursing home. However, by unknown cause and unknown to the COPI, one bus transported unharmed clients to the primary sheltering home of the city council.

At 08:40 a.m., the incident was upgraded to a GRIP II situation. A Regional Operational Team was formed, that consisted of the supreme commanders of all response units, members of the regional organization for medical assistance during accidents and disasters (GHOR) and supporting staff. The COPI advised the Regional Operational Team to improve external communication about the incident and to arrange psycho-social aftercare.

At 09:00 a.m., the incident was upgraded to a GRIP III situation by involvement of the mayor of Nieuwegein who-with a small municipal policy team-started the community disaster plan. The mayor and his team activated the cities sheltering location, started to communicate with the community and activated the Central Registration and Information Bureau for registration and identification of victims. However, the COPI and the nursing home had already taken care of many of these issues. Therefore, work was performed twice which initially confused some of the chain partners. 
Table 3 Hospital alert plan and response

\begin{tabular}{llllll}
\hline $\begin{array}{l}\text { Name of } \\
\text { hospital }\end{array}$ & $\begin{array}{l}\text { Distance from the } \\
\text { scene }(\mathrm{km})\end{array}$ & $\begin{array}{l}\text { Hospital } \\
\text { alerted }\end{array}$ & $\begin{array}{l}\text { Disaster plan } \\
\text { available }\end{array}$ & $\begin{array}{l}\text { Disaster plan } \\
\text { activated }\end{array}$ & $\begin{array}{l}\text { Receiving first } \\
\text { patient }\end{array}$ \\
\hline Hospital A & 2.7 & Yes & Yes & Yes & $08: 48$ \\
$\begin{array}{l}\text { Hospital B } \\
\text { Major Incident }\end{array}$ & 9.0 & No & Yes & No & - \\
$\begin{array}{l}\text { Hospital } \\
\text { Hospital D }\end{array}$ & 22.1 & Yes & Yes & Yes & $08: 53$ \\
Hospital E & 30.5 & No & Yes & No & - \\
\hline
\end{tabular}

Table 4 Coordination and command

\begin{tabular}{lll}
\hline Unit & \multicolumn{2}{l}{$\begin{array}{l}\text { Organization for coordination and } \\
\text { command }\end{array}$} \\
\cline { 2 - 3 } & Was prepared & Function score \\
\hline Ambulance & Yes & 5 \\
Helicopters & Yes & 1 \\
Pre-hospital medical teams & Yes & 1 \\
Hospitals & Yes & 5 \\
Rescue/civil protection & Yes & 5 \\
Police & Yes & 5 \\
Military & Yes & 1 \\
\hline
\end{tabular}

1 Never alerted, 2 alerted but did not function, 3 did function but needs major improvements, 4 did function, need minor improvements, 5 did function well

The MIH was activated through the correct hierarchical steps as described in the MIH protocol. The joint dispatch center requested the emergency department of the University Medical Center of Utrecht to activate the MIH. After consultation with the board of directors, the surgeon on call activated the MIH at 0834 hours (Fig. 1). Subsequently, all ambulances were (re)directed to the MIH. The operational command of the MIH consisted of the surgeon on call, the manager of the MIH and the manager of the emergency department. No major problems occurred in the coordination and command structure. Communication was unhampered between the operational command and the board of directors.

\section{Hospital damage}

No damage occurred to the MIH or to the hospital closest to the incident.

\section{Communication system (Table 5)}

No major communication or telephone issues arose during the initial phase of fire extinguishing and evacuation. The COPI was quickly operational and functioned properly. Because the COPI were tackling the first phase of the incident, communication between the COPI and the Major
Incident Coordinator (located in the joint emergency dispatch center) was suboptimal. Therefore, the Major Incident Coordinator was unable to obtain a complete impression of the situation. After 09:25 a.m., according to the report from the Institute for Safety and Crisis Management, the communication between COPI and the Regional Operational Team was constructive.

The National Crisis Management System, which is a relative new digital system, became active during this MCI. This online system receives input from any level of disaster management and can be used by any level (local emergency services, COPI, Regional Operational Team and municipal team). This system was used by the COPI and Regional Operational Team and not by the municipal policy team because it had not been fully implemented. In general, the system functioned in the way intended: producing an overview of the incident. However, although only relevant and verified information should be added, during this incident also partially verified facts, questions and uncertainties had been entered.

The local website of the Nieuwegein community displayed the first information about the incident at 1004 hours and the twitter-account of the community Nieuwegein was activated at 1015 hours. A special phone number was activated to answer questions from the community. Press spokesmen of the city council were sent to all shelter locations and a press conference was held by the municipal policy team at 1145 hours and by the MIH at 1600 hours. In the aftermath of the incident the municipal policy team informed the public through letters, press releases and group gatherings.

The use of the Patient Barcode and Register System in the MIH functioned well. In-ear walkie-talkies were used by all key staff members and one-way pagers and internal and external phones were used without major problems.

The location of each individual evacuee remained unclear for a prolonged time. Even though the majority of injured patients were transported to the $\mathrm{MIH}$, the registration and tracking of all (un)harmed nursing home clients and staff did not function properly. The GHOR requested personal information about patients admitted in the MIH; however, not all victims were able to identify themselves at 
Fig. 1 Flowchart of activation of the MIH. JED Joint Emergency Dispatch Center, $M M$ Medical Manager Trauma surgeon on call UMCU, $M I H$

Major Incident Hospital, $S N$

Senior Nurse Emergency

Department, UMCU University

Medical Center Utrecht
08:00 Media reported major fire

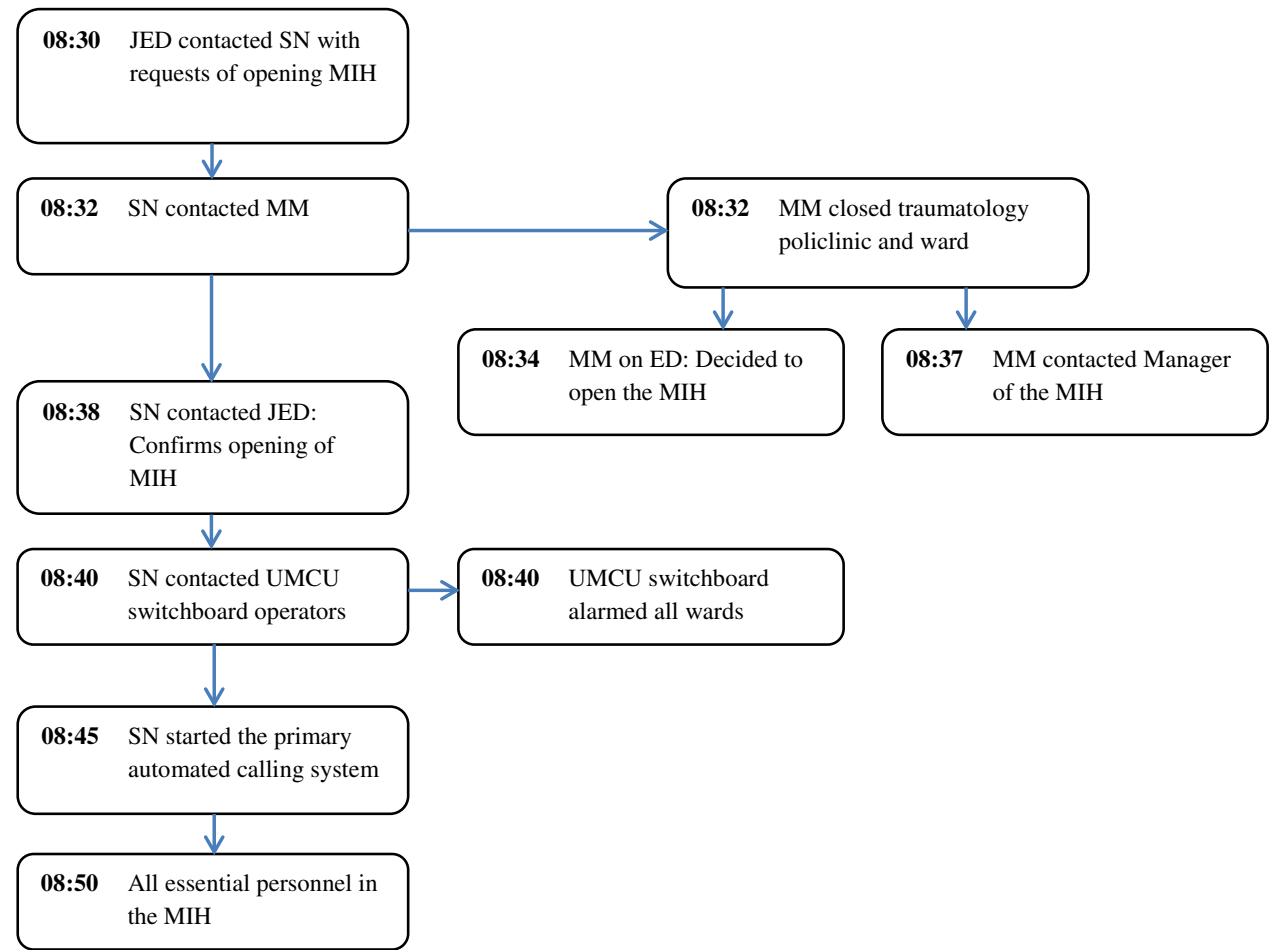

08:53 First patient in the MIH
Table 5 Communication systems

\begin{tabular}{lll}
\hline Unit & \multicolumn{2}{l}{ System and function score } \\
\cline { 2 - 3 } & $\begin{array}{l}\text { External } \\
\text { telephone net }\end{array}$ & $\begin{array}{l}\text { Internal } \\
\text { telephone net }\end{array}$ \\
\hline Ambulance & 3 & 3 \\
Helicopter & 0 & 0 \\
Pre-hospital teams & 0 & 0 \\
Hospitals & 3 & 3 \\
Rescue/civil protection & 3 & 3 \\
Police & 3 & 3 \\
Military & 3 & 3
\end{tabular}

0 Was not used, 1 did not function, 2 did function up to a point, 3 , did function well that time. Furthermore, the MIH managers were uncertain about the legal status of sharing (semi) medical information. A group of the nursing home staff members (22-25 persons) were allowed into the MIH for patient identification. Communication between the MIH and GHOR improved during the incident after a slow start.

Computer technology and back-up systems (Table 6)

No computer system failures occurred during the deployment of the MIH. The Patient Barcode and Register System of the MIH are easy to use and do not need extensive training. However, when the data were analyzed, it was discovered that the internal clock of the computer used for patient registration had not been set correctly. This led to a 
Table 6 Completeness of medical files during a MCI

\begin{tabular}{llll}
\hline & $\begin{array}{l}\text { T1 patients } \\
(\%)\end{array}$ & $\begin{array}{l}\text { T2 Patients } \\
(\%)\end{array}$ & $\begin{array}{l}\text { T3 Patients } \\
(\%)\end{array}$ \\
\hline $\begin{array}{l}\text { Number } \\
\text { History and current complaints }\end{array}$ & 11 & 29 \\
Allergy & $0(0)$ & $2(18.2)$ & $9(31)$ \\
Medical history & $1(16.7)$ & $4(36.4)$ & $15(51.7)$ \\
Home medication & $0(0)$ & $2(18.2)$ & $13(44.8)$ \\
Current complaint & $1(16.7)$ & $3(27.3)$ & $13(44.8)$ \\
Physical examination & & & \\
Airway & $0(0)$ & $8(72.7)$ & $24(82.8)$ \\
Breathing & $0(0)$ & $5(45.6)$ & $24(82.8)$ \\
Circulation & $0(0)$ & $4(36.4)$ & $11(37.9)$ \\
$\begin{array}{l}\text { Disability } \\
\text { Environment }\end{array}$ & $0(0)$ & $2(18.2)$ & $11(37.9)$ \\
Vital signs & $0(0)$ & $2(18.2)$ & $11(37.9)$ \\
Conclusion and recordkeeping & $6(33.3)$ & $6(54.5)$ & $24(82.8)$ \\
Conclusion and & $1(16.7)$ & $3(27.3)$ & $4(13.8)$ \\
$\quad$ treatment & & $3(27.3)$ & $12(41.4)$ \\
Recordkeeping by & $0(0)$ & $2(18.2)$ & $4(13.8)$ \\
$\quad$ nurses & & & \\
Recordkeeping by & $0(0)$ & & \\
physicians & & & \\
\hline
\end{tabular}

discrepancy in the registration time of the MIH and the unloading time documented by the ambulances.

Written patient charts were used to record patient data and all medical files were evaluated afterwards. Due to the high patient flow information had been poorly documented in the primary patient charts, especially of the $\mathrm{T} 1$ classed patients. For all transferred patients a discharge letter was prepared. Table 7 shows how data had been recorded in the primary patient chart.

Total number, type and severity of injuries (Fig. 2; Tables 7,8)

A total of 49 patients were treated in a hospital and medical data of all 46 patients admitted in the MIH were available for this study (Fig. 2).

In the MIH, the field triage was reassessed by an experienced surgeon. Thereafter, patients were transported to the corresponding triage- and treatment bay. The majority of patients had been exposed to soot and smoke. Only one patient had suffered from additional traumatic injury due to a difficult rescue procedure. Patients with imminent respiratory insufficiency caused by (possible) inhalation injury or soot in the larynx were intubated preemptively. In total, $6 \mathrm{~T} 1,11 \mathrm{~T} 2$ and $27 \mathrm{~T} 3$ patients were presented in the first $2 \mathrm{~h}$ after the incident. Two other T3 patients presented themselves later that day at the
Table 7 Patient characteristics, seen in the MIH

\begin{tabular}{|c|c|c|c|}
\hline & T1 patients & $\mathrm{T} 2$ patients & T3 patients \\
\hline Number & 6 & 11 & 29 \\
\hline Male/female & $1 / 5$ & $4 / 7$ & $16 / 13$ \\
\hline Age in years (limits) & $83(51-96)$ & $80(17-92)$ & $54(20-93)$ \\
\hline Above 50 years $(\%)$ & $6(100)$ & $8(82.7)$ & $14(48.3)$ \\
\hline Above 65 years $(\%)$ & $4(66.7)$ & $8(72.7)$ & $7(24.1)$ \\
\hline $\begin{array}{l}\text { Arrived in MIH after } \\
\text { alarm (min) }\end{array}$ & 58-107 & $53-156$ & $80-203^{\mathrm{a}}$ \\
\hline Intubated & 6 & 3 & 0 \\
\hline Admission & 6 & 8 & 4 \\
\hline IC UMCU & 2 & 2 & - \\
\hline $\begin{array}{l}\text { IC elsewhere in the } \\
\text { Netherlands }\end{array}$ & 4 & 1 & - \\
\hline Ward & - & 5 & 4 \\
\hline Discharge & - & 3 & 25 \\
\hline $\begin{array}{l}\text { Mean length of } \\
\text { stay (h) }\end{array}$ & $\begin{array}{l}3.10 \\
\quad(0.53-7.02)\end{array}$ & $\begin{array}{l}4.33 \\
\quad(2.42-6.16)\end{array}$ & $\begin{array}{l}5.17 \\
\quad(4.26-6.34)^{2}\end{array}$ \\
\hline
\end{tabular}

emergency department of the University Medical Centre of Utrecht. The median age of all 46 patients was 54 (17-96) years. Twenty-eight patients $(60.9 \%)$ were 50 years or older.

The first MCI patient was transported to the hospital closest to the incident with possible inhalation injury. Two others were transported later that day from the same incident address to this hospital, one in pulmonary distress, the other with a vascular occlusion, and it remains unknown if these patients were MCI patients.

\section{Initial treatment}

Six T1 patients arrived at the MIH within a range of 58-107 min after the initial alarm, their median age was 83 (51-96) years. After primary survey and stabilization, these six patients were directly intubated and transferred to the ICU of the MIH for further treatment.

Eleven $\mathrm{T} 2$ patients arrived at the MIH within a range of 53-156 min after the initial alarm, their median age was 80 (17-92) years. Two T2 patients needed mechanical ventilation directly after primary survey. Seven $\mathrm{T} 2$ patients were admitted to the medium care and two to the low care ward. Later that day, two T2 patients initially admitted on the medium care deteriorated due to inhalation trauma; both were admitted on the ICU, one needed mechanical ventilation.

Twenty-seven T3 patients arrived at the MIH by ambulance 80-203 min after the initial alarm, median age 54 (20-93) years. Two patients reported themselves later at 
Fig. 2 Patient flowchart

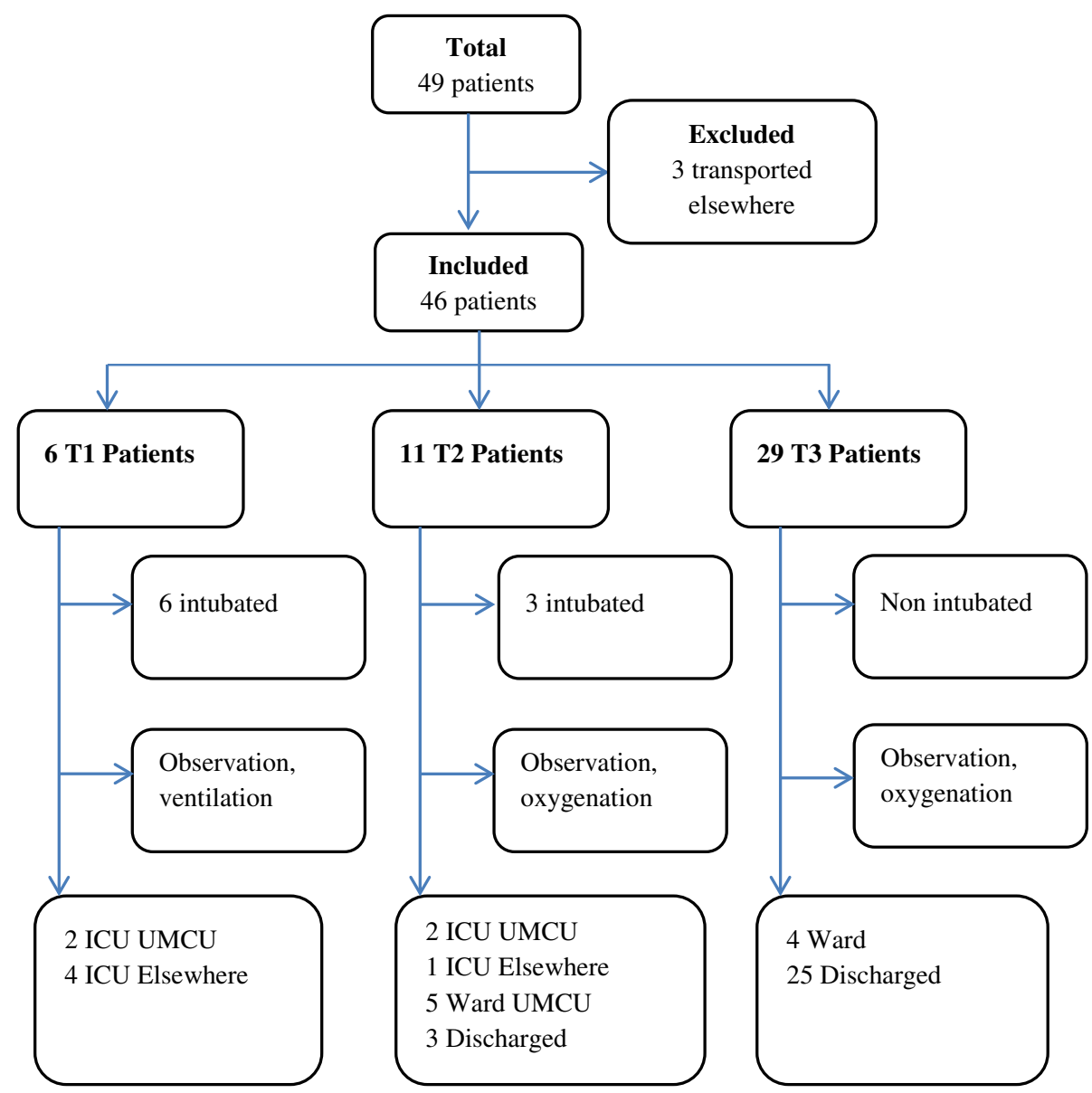

Table 8 Severity of injuries according to treatment

\begin{tabular}{lll}
\hline Type & $\begin{array}{l}\text { Initial } \\
\text { survey }\end{array}$ & $\begin{array}{l}\text { After primary } \\
\text { observation }\end{array}$ \\
\hline $\begin{array}{l}\text { Less severe injuries not examined or } \\
\text { treated by medical professionals }\end{array}$ & 197 & Unknown \\
$\begin{array}{l}\text { Less to moderately severe injuries } \\
\text { examined an or treated by medical } \\
\text { professionals }\end{array}$ & 49 & - \\
$\begin{array}{l}\text { Outside hospital } \\
\text { In hospital }\end{array}$ & Unknown & - \\
$\begin{array}{l}\text { Injuries requiring in-hospital care/ } \\
\text { observation in the MIH }\end{array}$ & 49 & 27 \\
$\begin{array}{l}\text { In a regular ward } \\
\text { In intensive care }\end{array}$ & 37 & 9 \\
Requiring a ventilator & 9 & 9 \\
Injuries requiring surgery & 9 & 9 \\
\hline
\end{tabular}

the emergency department of the UMCU and were linked to the MIH database $356 \mathrm{~min}$ after the initial alarm. The T3 patients were observed in the low care ward and were given oxygen.

\section{Transferal from the $M I H$}

Overall, nine patients had needed intubation and mechanical ventilation. After a mean length of stay of $3.10 \mathrm{~h}$, median $2.13(0.53-7.02) \mathrm{h}$, four had been admitted to the ICU of the UMCU hospital and five had been transported to ICU's elsewhere in the Netherlands. After a median length of stay of $4.22(2.42-6.16) \mathrm{h}$, nine patients were transferred to a ward in the UMCU for one night observation to exclude delayed onset of pulmonary symptoms. Twenty-six patients could be discharged directly from the MIH after median length of stay of 5.45 (4.26-6.19) h. One of the self-referred patients was transferred to a nearby hospital for further observation; the other patient did not need further observation.

Hospital load

Congestion or lack of material or resources did not occur since the MIH is in essence a stand-alone hospital with a separate stock of supplies. If needed, the MIH can resupply through the UMCU and the Central Military Hospital. The 
maximum surge capacity of the MIH is 60 patients per hour (with a maximum of $16 \mathrm{~T} 1$ patients, $24 \mathrm{~T} 2$ patients per hour). These limits were never approached with the 46 admissions, (6 T1, $11 \mathrm{~T} 2$ and $29 \mathrm{~T} 3$ patients) that were presented in $4 \mathrm{~h}$.

Approximately 175 staff members had been deployed from the University Medical Center Utrecht and the adjoining Central Military Hospital of which $\sim 110$ nurses and physicians and 65 supporting staff members. For security reasons, all personnel had passed the registration counter. However, the information on the (handwritten) registration forms was often incomplete or unreadable.

Psychological reactions

Following the MIH activation protocol staffs from the psychology and psychiatric departments were alerted. They have a key role in the direct psychological aftercare for victims, family and if needed medical staff members.

After the incident, group- and individual counseling sessions were organized for all personnel and clients primarily through the Dutch Victim Support group. Moreover, the nursing home itself enabled group- and individual counseling sessions with psychologists specialized in crises related trauma.

\section{Outcome}

No pre- or in-hospital deaths occurred. After 3 months, no deaths or persistent disabilities could be linked to the incident. Some nursing home staff members had psychologically suffered from the fire, resulting in frightened reactions after every new fire alarm.

Estimated number of people affected but not injured

Of the 138 inhabitants and 49 staff members of the nursing home, 49 had needed primary medical attention after the incident. Another 31 patients could be discharged after primary observation and treatment in the MIH. The 139 unharmed clients and staff members were temporarily sheltered elsewhere.

\section{Post-accident evaluation (Table 9)}

\section{Post-accident evaluation of preparedness}

In general the MIH functioned according to the disaster plan. Directly after the incident, all staff members could vent their opinion on every aspect of the MIH activation. This was captured in the first impression report. In the following days a questionnaire was sent to all participating staff members. An evaluation report was drawn up based upon the first impression report, questionnaire and personal experiences. Some areas that need improvement will be addressed in the discussion.

\section{Post-accident evaluation of training}

During the deployment it appeared that the majority of (key) staff members had been trained properly. Key staff members had been assigned coordinating roles and helped others who were less familiar with the MIH procedures. However, despite monthly offering of training, a minority of non-key staff members were not familiar with the MIH procedures.

\section{Discussion}

This report describes the medical response to a MCI caused by a fire in a nursing home. In total, 49 persons needed medical treatment in a hospital. No deaths occurred during the incident or in the 3 months afterwards.

The protocol for reports from major accidents and disasters [1] was used to uniform data reporting. A systematic review compared different available protocols of standardized reporting [14] and we chose this protocol

Table 9 Post accident evaluation of the quality of preparedness and of training

\begin{tabular}{llll}
\hline Organization & \multicolumn{2}{l}{ Accuracy of disaster plan } & \\
\cline { 2 - 4 } & Disaster plan existed & Plan activated & Functioning of disaster plan \\
\hline Hospital A & Yes & Yes & Unknown \\
Major Incident Hospital & Yes & Yes & Did function in most parts, but needs minimal revisions \\
\hline Organization & Post-accident evaluation of training & \\
\cline { 2 - 4 } & Had any training or exercise been done? & Extent of accuracy of training \\
\hline Hospital A & Unknown & Unknown \\
Major Incident Hospital & Yes & Extent of training was sufficient and methodology was accurate
\end{tabular}


because it was developed to report real incidents, has a practical approach, is freely available, has been published $[1,15,16]$ and has been used previously [10, 17, 18]. Other protocols are more extensive but less practical, such as the Utstein template [19], the DISAST-CIR, a protocol used by the Israeli Defense Force Home Front and Ministry of Health [20-28] and two unused protocols [29, 30]. None of these protocols have been tested on Internal and external validity [14].

In the pre-hospital phase of the current incident, the different levels of incident managers performed a tight coordination and all emergency services had arrived quickly at the scene. Transport resources were sufficient and were made possible through the support of neighboring safety regions. The decision to activate the MIH was based on the regional disaster plan and had the immediate advantage of being an easy accessible hospital with sufficient capacity to treat all injured patients. In hindsight, the activation of the disaster plan by the nearest hospital might have been unnecessary since nearly all patients were transported to the MIH. However, initially it was unclear if the MIH would open and previous studies have shown that hospitals closest to the incident were overrun by selfreferrers [8, 31] and emergency medical services [32, 33].

In the pre-hospital phase two major areas of improvement could be recognized. First due to miscommunication between the different chain partners (local incident commanders, joint emergency dispatch center, the Regional Operational Team and municipal policy team), they were not aware what other chain partners had already accomplished. The communication could be ameliorated if all partners use the National Crisis Management System. If used correctly, this system produces an accurate and immediate overview of all aspects of the MCI and will facilitate interdisciplinary communication between the incident site and chain partners. For instance, if the municipal policy team had been informed that sheltering homes, registration of (unharmed) victims and the aftercare were already arranged by the local incident commanders, they could have focused on other matters.

Second, the registration and tracking of victims proved to be troublesome. Both the local incident commanders and the municipal policy team had arranged sheltering locations. Unknown to the incident commanders, unharmed and unregistered clients were sent to the city councils primary sheltering location. Thereby, it became unclear who was transported to which sheltering home or hospital. Tracking victims during a MCI is a recurrent problem in the Netherlands which is depicted in ministerial reports after the Volendam Café Fire (2001), Queensday incident (2009), Airplane crash (2009) and Shooting incident (2011). The problem often arises due to the lack of reliable and fast technical regional or national systems to register patients before transportation. In 2005 a Victim Tracking System was developed by the UMCU, Regional Ambulance Service Utrecht and GHOR Utrecht which proved its value in registration of victims and enables patient tracking and tracing [34]. However, this system was not implemented in the Netherlands. A (national) tracking system will improve quick registration and triage. Receiving hospitals can anticipate on the number and type of patients en route. And it generates an overview of total patients and type of patients for any level of incident managers.

During the current incident, the MIH had been activated through the correct hierarchical steps and functioned properly. The high number of (pre-emptive) intubations might be considered as overtreatment. However, all of the intubated patients displayed one or more physical findings suggesting respiratory tract injury. Except for the Volendam fire [35], in nearly all previous described indoor fires, most deaths were caused by inhalation injury or high concentration of toxics and less due to severe burns [3643]. Additionally, the intubated patients were of older age, which is an independent major risk factor for mortality [44-48].

Some major areas for improvement could be discerned in the hospital phase. First, only the primary automated calling system was used, calling key staff members only. Also, this system could not reach all key staff on their mobile phones at that particular hour of day. Switchboard operators reached them eventually; however, this took a considerable amount of time. Because the UMCU was already in full service, all other personnel were recruited from wards and the out-patient departments. Initially, the ICU of the MIH was understaffed. Staffing of the ICU and the amount of mechanical ventilators are always potential bottlenecks during a MCI [49-51]. Using the secondary calling system would have alarmed more ICU staff members as well as volunteers of the Dutch Red Cross, resulting in more helping hands. In future activations of the MIH, staff shortages can be prevented by invariably initiating the secondary calling system. To facilitate the entire warning process new ideas are currently implemented, using a new automated warning system that can place automated internal calls and send mobile text messages. In case of activation of the MIH, every UMCU and Central Military Hospital computer should receive an informative pop-up.

Second, even though the MIH offers a monthly training for all staff, it became clear that not all UMCU personnel were familiar with the procedures of the MIH. However, the outcome was not influenced since proper trained key staff members had coordinating roles. Nevertheless, efforts to increase knowledge of $\mathrm{MIH}$ organizational procedures for non-key staff members will be intensified.

Third, the handwritten forms to register incoming personnel proved incomplete or unreadable. This led to the 
prompt development of an automated system through which personnel can register themselves by swiping their employee card in front of a radio frequency identification reader. A hospital security member will ensure that every staff member is registered by the scanner.

Fourth, the written medical charts proved to be too elaborate for an acute deployment. The initial registration of patient data during the primary survey was very poor. Therefore, a new concise medical file has been developed which already proved its value in a recent major exercise and during other activations of the MIH.

Fifth, sharing (semi) medical information between hospitals and the medical assistance during accidents and disasters and governmental officials is a recurrent problem in the Netherlands as also concluded by the ministerial reports after the Volendam Café Fire (2001), Queensday incident (2009), Airplane crash (2009) and Shooting incident (2011) and independent research [52]. During the current incident, the MIH did not release this information directly to the responsible body due to the pressure of increased patient flow, the impossibility to identify patients directly and partly due to the unfamiliarity with the legal status. New covenants between the medical assistance during accidents and disasters and hospitals have recently been signed to avoid these uncertainties in the future.

\section{Conclusion}

The pre-hospital (medical) services, coordinating bodies, and medical assistance during accidents and disasters performed fast and good work. Despite the fact that the majority of patients were elderly and/or disabled, no pre- or in-hospital deaths occurred. Furthermore in the 3 months after the MCI, no deaths or persistent disabilities could be linked to the incident. The MIH demonstrated its strength to provide emergency care for 46 patients and was able to quickly accommodate nine intubated patients. In the prehospital phase, communication between chain partners and victim tracking should be improved. In the MIH, improvements will be implemented in the calling system, registration of incoming staff, training of non-key staff members, the development of a concise medical chart and improve communication between the chain patterns.

\begin{abstract}
Acknowledgments The authors would like to thank nursing home "De Geinsche Hof" and specially Ms. M. de Graaf, coordinating location manager, for her open interview. We would also like to thank the Regional Ambulance Service Utrecht for their effort in retrieving detailed times of used ambulances. Finally we would like to thank the Institute of Safety and Crisis management for their detailed rapport.
\end{abstract}

Conflict of interest Salomon W. Koning, Pauline M. Ellerbroek, and Luke PH. Leenen declare that they have no conflict of interest.
Ethical statement The Medical Ethical Research Committee of the University Medical Center Utrecht reviewed the research protocol. The Committee granted a written approval that this study is within Dutch medical research law (Protocol Number 12-479/C). Privacy of all patients was maintained at all times. Medical files were stored in a locked cabinet and the data were stored on a password-protected computer.

\section{References}

1. Lennquist S. Protocol for reports from major accidents and disasters in the International Journal of Disaster Medicine. Int J Disaster Med. 2003;1(1):79-86.

2. al-Madhari AF, Keller AZ. Review of disaster definitions. Prehospital Disaster Med. 1997;12(1):17-20.

3. Koenig KL, Dinerman N, Kuehl AE. Disaster nomenclature-a functional impact approach: the PICE system. Acad Emerg Med. 1996;3(7):723-7.

4. Lennquist S. Management of major accidents and disasters: an important responsibility for the trauma surgeons. J Trauma. 2007;62(6):1321-9.

5. Lennquist $\mathrm{S}$. Medical response to major incidents and disasters: a practical guide for all medical staff. 1st ed. Heidelberg: Springer; 2012.

6. Einav S, Aharonson-Daniel L, Weissman C, Freund HR, Peleg K. In-hospital resource utilization during multiple casualty incidents. Ann Surg. 2006;243(4):533-40.

7. Frykberg ER. Triage: principles and practice. Scand J Surg. 2005;94(4):272-8.

8. Miller KT. Emergency medical services scene management. In: Koenig KL, Schultz CH, editors. Disaster medicine: comprehensive principles and practices. 1st ed. New York: Cambridge University Press; 2010.

9. Marres GMH, Bemelman M, van der Eijk J, Leenen LPH. Major Incident Hospital: development of a permanent facility for management of incidents casualties. Eur J Trauma Emerg Surg. 2009;35(3):203-11.

10. Marres GMH, van der Eijk J, Bemelman M, Leenen LPH. Evaluation of admissions to the Major Incident Hospital based on a standardized protocol. Eur J Trauma Emerg Surg. 2011;37(1):19-29.

11. Bouman JH, Schouwerwou RJ, Van der Eijk KJ, van Leusden AJ, Savelkoul TJ. Computerization of patient tracking and tracing during mass casualty incidents. Eur $\mathrm{J}$ Emerg Med. 2000;7(3):211-6.

12. Noordergraaf GJ, Bouman JH, van den Brink EJ, van de Pompe C, Savelkoul TJ. Development of computer-assisted patient control for use in the hospital setting during mass casualty incidents. Am J Emerg Med. 1996;14(3):257-61.

13. American Burn Association. Airway management and smoke inhalation injury. In: Advanced burn life support-provider manual. Digital ed. Chicago: 2007.

14. Fattah S, Rehn M, Reierth E, Wisborg T. Systematic literature review of templates for reporting prehospital major incident medical management. BMJ Open. 2013;3(8):e002658. doi:10. 1136/bmjopen-2013-002658.

15. Lennquist S. Protocol for reports from major accidents and disasters in the International Journal of Disaster Medicine. Int $\mathbf{J}$ Disaster Med. 2004;2:57-64.

16. Lennquist S. Protocol for reports from major accidents and disasters in the International Journal of Disaster Medicine. Eur J Trauma Emerg Surg. 2008;34(5):486-92.

17. Backman K, Albertsson P, Pettersson S, Björnstig U. Protocol from the coach crash in Ängelsberg, Sweden January 2003. Int J Disaster Med. 2004;2:93-104. 
18. Dami F, Fuchs V, Péclard E, Potin M, Vallotton L, Carron P. Coordination of emergency medical services for a major road traffic accident on a Swiss suburban highway. Eur J Trauma Emerg Surg. 2013;35(3):265-70.

19. Debacker M, Hubloue I, Dhondt E, Rockenschaub G, Ruter A, Codreanu $\mathrm{T}$, et al. Utstein-style template for uniform data reporting of acute medical response in disasters. PLoS Curr. 2012;4:e4f6cf3e8df15a.

20. Schwartz D, Pinkert M, Leiba A, Oren M, Haspel J, Levi Y, et al. Significance of a Level-2, "selective, secondary evacuation" hospital during a peripheral town terrorist attack. Prehospital Disaster Med. 2007;22(1):59-66.

21. Leiba A, Schwartz D, Eran T, Blumenfeld A, Laor D, Goldberg A, et al. DISAST-CIR: disastrous incidents systematic analysis through components, interactions and results: application to a large-scale train accident. J Emerg Med. 2009;37(1):46-50.

22. Schwartz D, Ostfeld I, Bar-Dayan Y. A single, improvised "Kassam" rocket explosion can cause a mass casualty incident: a potential threat for future international terrorism? Emerg Med J. 2009;26(4):293-8.

23. Pinkert M, Lehavi O, Goren OB, Raiter Y, Shamis A, Priel Z, et al. Primary triage, evacuation priorities, and rapid primary distribution between adjacent hospitals-lessons learned from a suicide bomber attack in downtown Tel-Aviv. Prehospital Disaster Med. 2008;23(4):337-41.

24. Schwartz D, Bar-Dayan Y. Injury patterns in clashes between citizens and security forces during forced evacuation. Emerg Med J. 2008;25(10):695-8.

25. Lehavi O, Leiba A, Dahan Y, Schwartz D, Benin-Goren O, Schwartz R, et al. Lessons learned from chlorine intoxications in swimming pools: the challenge of pediatric mass toxicological events. Prehospital Disaster Med. 2008;23(1):90-5.

26. Raiter Y, Farfel A, Lehavi O, Goren OB, Shamiss A, Priel Z, et al. Mass casualty incident management, triage, injury distribution of casualties and rate of arrival of casualties at the hospitals: lessons from a suicide bomber attack in downtown Tel Aviv. Emerg Med J. 2008;25(4):225-9.

27. Bloch YH, Schwartz D, Pinkert M, Blumenfeld A, Avinoam S, Hevion G, et al. Distribution of casualties in a mass-casualty incident with three local hospitals in the periphery of a densely populated area: lessons learned from the medical management of a terrorist attack. Prehospital Disaster Med. 2007;22(3):186-92.

28. Pinkert M, Leiba A, Zaltsman E, Erez O, Blumenfeld A, Avinoam $\mathrm{S}$, et al. The significance of a small, level-3 'semi evacuation' hospital in a terrorist attack in a nearby town. Disasters. 2007;31(3):227-35.

29. Kulling P, Birnbaum M, Murray V, Rockenschaub G. Guidelines for reports on health crises and critical health events. Prehospital Disaster Med. 2010;25(4):377-83.

30. Ricci E, Pretto E. Assessment of prehospital and hospital response in disaster. Crit Care Clin. 1991;7(2):471-84.

31. Auf der Heide E. The importance of evidence-based disaster planning. Ann Emerg Med. 2006;47(1):34-49.

32. Hogan DE, Waeckerle JF, Dire DJ, Lillibridge SR. Emergency department impact of the Oklahoma City terrorist bombing. Ann Emerg Med. 1999;34(2):160-7.

33. Okumura T, Takasu N, Ishimatsu S, Miyanoki S, Mitsuhashi A, Kumada K, et al. Report on 640 victims of the Tokyo subway sarin attack. Ann Emerg Med. 1996;28(2):129-35.
34. Marres GM, Taal L, Bemelman M, Bouman J, Leenen LP. Online victim tracking and tracing system (ViTTS) for major incident casualties. Prehospital Disaster Med. 2013;28(5):445-53.

35. Welling L, van Harten SM, Patka P, Bierens JJ, Boers M, Luitse JS, et al. The cafe fire on New Year's Eve in Volendam, the Netherlands: description of events. Burns. 2005;31(5):548-54.

36. Allister C, Hamilton GM. Cardowan coal mine explosion: experience of a mass burns incident. Br Med J (Clin Res Ed). 1983;287(6389):403-5.

37. O'Hickey SP, Pickering CA, Jones PE, Evans JD. Manchester air disaster. Br Med J (Clin Res Ed). 1987;294(6588):1663-7.

38. Ramos G, Flageat G, Queiroz G, Nacif G, Fiorentino R, Arata A, et al. Massive hospital admission of patients with respiratory failure resulting from smoke inhalation injury: the Cromagnon Republic Tragedy. J Burn Care Res. 2006;27(6):842-7.

39. Saffle JR. The 1942 fire at Boston's Cocoanut Grove nightclub. Am J Surg. 1993;166(6):581-91.

40. Greenhalgh DG, Chang P, Maguina P, Combs E, Sen S, Palmieri TL. The ABC daycare disaster of Hermosillo, Mexico. J Burn Care Res. 2012;33(2):235-41.

41. Cassuto J, Tarnow P. The discotheque fire in Gothenburg 1998. A tragedy among teenagers. Burns. 2003;29(5):405-16.

42. Buerk CA, Batdorf JW, Cammack KV, Ravenholt O. The MGM Grand Hotel fire: lessons learned from a major disaster. Arch Surg. 1982;117(5):641-4.

43. Hart RJ, Lee JO, Boyles DJ, Batey NR. The Summerland disaster. Br Med J. 1975;1(5952):256-9.

44. Brusselaers N, Monstrey S, Vogelaers D, Hoste E, Blot S. Severe burn injury in Europe: a systematic review of the incidence, etiology, morbidity, and mortality. Crit Care. 2010;14(5):R188.

45. Coste J, Wasserman D, Venot A. Predicting mortality in adult burned patients: methodological aspects of the construction and validation of a composite ratio scale. J Clin Epidemiol. 1996;49(10):1125-31.

46. Galeiras R, Lorente JA, Pertega S, Vallejo A, Tomicic V, de la Cal MA, et al. A model for predicting mortality among critically ill burn victims. Burns. 2009;35(2):201-9.

47. Germann G, Barthold U, Lefering R, Raff T, Hartmann B. The impact of risk factors and pre-existing conditions on the mortality of burn patients and the precision of predictive admission-scoring systems. Burns. 1997;23(3):195-203.

48. The Belgian Outcome in Burn Injury Study Group. Development and validation of a model for prediction of mortality in patients with acute burn injury. Br J Surg. 2009;96(1):111-7.

49. Hick JL, Rubinson L, O'Laughlin DT, Farmer JC. Clinical review: allocating ventilators during large-scale disasters-problems, planning, and process. Crit Care. 2007;11(3):217.

50. Neyman G, Irvin CB. A single ventilator for multiple simulated patients to meet disaster surge. Acad Emerg Med. 2006;13(11):1246-9.

51. Singer AJ, Singer AH, Halperin P, Kaspi G, Assaf J. Medical lessons from terror attacks in Israel. $\mathrm{J}$ Emerg Med. 2007;32(1):87-92.

52. Postma IL, Winkelhagen J, Bloemers FW, Heetveld MJ, Bijlsma TS, Goslings JC. February 2009 airplane crash at Amsterdam Schiphol airport: an overview of injuries and patient distribution. Prehospital Disaster Med. 2011;26(4):299-304. 